\title{
Acute myeloblastic leukemia without maturation
}

INSERM

\section{Source}

INSERM. (1999). Orphanet: an online rare disease and orphan drug data base. Acute myeloblastic leukemia without maturation. ORPHA:98833

Acute myeloblastic leukemia without maturation is a rare, acute myeloid leukemia characterized by no significant myeloid maturation and more than $90 \%$ blast cells in the non-erythroid population. Various degrees of anemia, thrombocytopenia, or pancytopenia are present. Frequent clinical manifestations include fatigue, fever, bleeding disorders, and organomegaly, especially hepatosplenomegaly. 